\title{
On some Hadamard-type inequalities for product of two s-convex functions on the co-ordinates
}

\author{
Muhamet Emin Özdemir ${ }^{1}$, Muhammad Amer Latif ${ }^{2}$ and Ahmet Ocak Akdemir ${ }^{3 *}$
}

* Correspondence:

ahmetakdemir@agri.edu.tr

${ }^{3}$ Department of Mathematics,

Faculty of Science and Letters, A $\breve{g r i}$

İbrahim Çeçen University, A $\breve{g r i}$

04100, Turkey

Full list of author information is available at the end of the article

\section{Abstract}

In this article, Hadamard-type inequalities for product of s-convex in the second sense on the co-ordinates in a rectangle from the plane are established.

\section{Introduction}

A function $f: I \rightarrow \mathbb{R}, I \subseteq \mathbb{R}$ is an interval, is said to be a convex function on $I$ if

$$
f(t x+(1-t) y) \leq t f(x)+(1-t) f(y)
$$

holds for all $x, y \in I$ and $t \in[0,1]$. If the reversed inequality in (1.1) holds, then $f$ is concave. Let $f: I \subseteq \mathbb{R} \rightarrow \mathbb{R}$ be a convex function and $a, b \in I$ with $a<b$. Then the following double inequality:

$$
f\left(\frac{a+b}{2}\right) \leq \frac{1}{b-a} \int_{a}^{b} f(x) d x \leq \frac{f(a)+f(b)}{2}
$$

is known as Hermite-Hadamard inequality for convex mappings. For particular choice of the function $f$ in (1.2) yields some classical inequalities of means. Both inequalities in (1.2) hold in reversed direction if $f$ is concave.

Some basic definitions can be given as followings:

Definition 1. (See [1], [2, p. 410]) We say that $f: I \rightarrow \mathbb{R}$ is a Godunova-Levin function or that $f$ belongs to the class $Q(I)$ if $f$ is non-negative and for all $x, y \in I$ and $t \in$ $(0,1)$ we have

$$
f(t x+(1-t) y) \leq \frac{f(x)}{t}+\frac{f(y)}{1-t}
$$

Definition 2. (See [3]) We say that $f: I \subseteq \mathbb{R} \rightarrow \mathbb{R}$ is a P-function or that $f$ belongs to the class $P(I)$ if $f$ is non-negative and for all $x, y \in I$ and $t \in[0,1]$, we have

$$
f(t x+(1-t) y) \leq f(x)+f(y) .
$$

Definition 3. (See [4]) Let $s \in(0,1]$. A function $f:[0, \infty) \rightarrow[0, \infty)$ is said to be $s$ convex in the second sense if

$$
f(t x+(1-t) y) \leq t^{s} f(x)+(1-t)^{s} f(y)
$$

for all $x, y \in(0, b)$ and $t \in[0,1]$.

\section{Springer}

(c) 2012 Özdemir et al; licensee Springer. This is an Open Access article distributed under the terms of the Creative Commons Attribution License (http://creativecommons.org/licenses/by/2.0), which permits unrestricted use, distribution, and reproduction in any medium, provided the original work is properly cited. 
In [5], Hudzik and Maligranda considered among others the class of functions which are $s$-convex in the first sense. This class is defined in the following way:

Definition 4. A function $f: \mathbb{R}^{+} \rightarrow \mathbb{R}$, where $\mathbb{R}^{+}=[0, \infty)$, is said to be s-convex in the first sense if

$$
f(\alpha x+\beta y) \leq \alpha^{s} f(x)+\beta^{s} f(y)
$$

for all $x, y \in[0, \infty), \alpha, \beta \geq 0$ with $\alpha^{s}+\beta^{s}=1$ and for some fixed $s \in(0,1]$. We denote by $K_{s}^{1}$ the class of all s-convex functions.

In 1978, Breckner introduced $s$-convex functions as a generalization of convex functions in [4]. Also, he proved the important fact that the set valued map is $s$-convex only if the associated support function is $s$-convex function [6]. Of course, $s$-convexity means just convexity when $s=1$. The definition of $s$-convexity of real valued functions are very important for Orlicz spaces and Banach normed spaces (see [7-9]). A number of properties of $s$-convex functions are discussed in articles [5,10-13].

In article [14] the following generalization of the previously described functions was given.

Definition 5. (See $[14,15])$ Let $I$, $J$ be intervals $\mathbb{R},(0,1) \subseteq J$ and let $h: J \rightarrow \mathbb{R}$. A function $f: I \rightarrow \mathbb{R}$ is called an $h$-convex function, or that $f$ belongs to the class $S X(h, I)$, if for all $x, y \in I$ and $t \in(0,1)$ we have

$$
f(t x+(1-t) y) \leq h(t) f(x)+h(1-t) f(y) .
$$

If inequality in (1.3) is reversed, then $f$ is said to be h-concave.

Obviously, if $h(t)=t$, for all $t \in[0,1] \subseteq J$, then all convex functions belong to $S X$ ( $h$, $I$ ) and all concave functions are $h$-concave; if $h(t)=\frac{1}{t}$, for all $t \in(0,1)$, then $S X(h, I)=$ $Q(I)$; if $h(t)=1, S X(h, I) \supseteq P(I)$; and if $h(t)=t^{s}$, where $s \in(0,1)$, then $S X(h, I) \supseteq K_{s}^{2}$. For some recent results about $h$-convex functions we refer the reader to articles [15-18].

In [10], Dragomir and Fitzpatrick proved the following variant of Hermite-Hadamard inequality which hold for $s$-convex functions in the second sense:

Theorem 1. Suppose that $f:[0, \infty) \rightarrow[0, \infty)$ is an s-convex function in the second sense, where $s \in(0,1)$ and let $a, b \in[0, \infty), a<b$. If $f \in L_{1}([a, b])$, then the following inequalities hold:

$$
2^{s-1} f\left(\frac{a+b}{2}\right) \leq \frac{1}{b-a} \int_{a}^{b} f(x) d x \leq \frac{f(a)+f(b)}{s+1}
$$

The constant $k=\frac{1}{s+1}$ is the best possible in the second inequality in (1.4).

Again in [10], Dragomir and Fitzpatrick also proved the following Hadamard-type inequality for $s$-convex functions in the first sense:

Theorem 2. Suppose that $f:[0, \infty) \rightarrow[0, \infty)$ is an s-convex function in the first sense, where $s \in(0,1)$ and let $a, b \in[0, \infty)$. If $f \in L_{1}([a, b])$ then the following inequalities hold:

$$
f\left(\frac{a+b}{2}\right) \leq \frac{1}{b-a} \int_{a}^{b} f(x) d x \leq \frac{f(a)+s f(b)}{s+1}
$$

The above inequalities are sharp. 
A modification for convex functions which is also known as co-ordinated convex functions was introduced as following by Dragomir [19]:

Let us consider a bidimensional interval $\Delta=:[a, b] \times[c, d]$ in $\mathbb{R}^{2}$ with $a<b$ and $c<$ d. A mapping $f: \Delta \rightarrow \mathbb{R}$ is said to be convex on $\Delta$ if the following inequality:

$$
f(\alpha x+(1-\alpha) z, \alpha y+(1-\alpha) w) \leq \alpha f(x, y)+(1-\alpha) f(z, w)
$$

holds, for all $(x, y),(z, w) \in \Delta$ and $\alpha \in[0,1]$.

A function $f: \Delta \rightarrow \mathbb{R}$ is said to be convex on the co-ordinates on $\Delta$ if the partial mappings $f_{y}:[a, b] \rightarrow \mathbb{R}, f_{y}(u)=f(u, y)$ and $f_{x}:[c, d] \rightarrow \mathbb{R}, f_{x}(v)=f(x, v)$ are convex where defined for all $x \in[a, b], y \in[c, d]$.

In the same article, Dragomir established the following Hadamard-type inequalities for convex functions on the co-ordinates in a rectangle from the plane $\mathbb{R}^{2}$ :

Theorem 3. Suppose $f: \Delta=[a, b] \times[c, d] \subseteq[0, \infty) \rightarrow \mathbb{R}$ is convex function on the coordinates on $\Delta$. Then one has the inequalities:

$$
\begin{aligned}
f\left(\frac{a+b}{2}, \frac{c+d}{2}\right) & \leq \frac{1}{(b-a)(d-c)} \int_{a}^{b} \int_{c}^{d} f(x, y) d y d x \\
& \leq \frac{f(a, c)+f(b, c)+f(a, d)+f(b, d)}{4}
\end{aligned}
$$

The concept of $s$-convex functions on the co-ordinates in both sense was introduced by Alomari and Darus [20,21]:

Definition 6. Consider the bidimensional interval $\Delta=:[a, b] \times[c, d]$ in $[0, \infty)^{2}$ with $a<b$ and $c<d$. The mapping $f: \Delta \rightarrow \mathbb{R}$ is s-convex in the first sense (in the second sense) on $\Delta$ if

$$
f(\alpha x+\beta z, \alpha y+\beta w) \leq \alpha^{s} f(x, y)+\beta^{s} f(z, w)
$$

holds for all $(x, y),(z, w) \in \Delta, \alpha, \beta \geq 0$ with $\alpha^{s}+\beta^{s}=1(\alpha+\beta=1)$ and for some fixed $s \in(0,1]$. We write $f \in K_{s}^{i}(i=1,2)$ which means that $f$ is $s$-convex in the first sense when $i=1$, (in the second sense when $i=2$ ).

A function $f: \Delta=:[a, b] \times[c, d] \subseteq[0, \infty)^{2} \rightarrow \mathbb{R}$ is called $s$-convex in first sense (in the second sense) on the co-ordinates on $\Delta$ if the partial mappings $f_{y}:[a, b] \rightarrow \mathbb{R}, f_{y}$ $(u)=f(u, y)$ and $f_{x}:[c, d] \rightarrow \mathbb{R}, f_{x}(v)=f(x, v)$, are $s$-convex in the first sense (in the second sense) for all $y \in[c, d], x \in[a, b]$, and $s \in(0,1]$, i.e, the partial mappings $f_{y}$ and $f_{x}$ are $s$-convex in the first sense (second sense) with some fixed $s \in(0,1]$.

In [20], Alomari and Darus proved the following inequalities for $s$-convex functions (in the second sense) on the co-ordinates in a rectangle from the plane $\mathbb{R}^{2}$ :

Theorem 4. Suppose $f: \Delta=[a, b] \times[c, d] \subseteq[0, \infty) \rightarrow \mathbb{R}$ is s-convex function (in the second sense) on the co-ordinates on $\Delta$. Then one has the inequalities:

$$
\begin{aligned}
4^{s-1} f\left(\frac{a+b}{2}, \frac{c+d}{2}\right) & \leq \frac{1}{(b-a)(d-c)} \int_{a}^{b} \int_{c}^{d} f(x, y) d y d x \\
& \leq \frac{f(a, c)+f(b, c)+f(a, d)+f(b, d)}{(s+1)^{2}}
\end{aligned}
$$

Also in [21] (see also [22]), Alomari and Darus established the following inequalities for $s$-convex functions (in the first sense) on the co-ordinates in a rectangle from the plane $\mathbb{R}^{2}$ : 
Theorem 5. Suppose $f: \Delta=[a, b] \times[c, d] \subseteq[0, \infty) \rightarrow \mathbb{R}$ is s-convex function (in the first sense) on the co-ordinates on $\Delta$. Then one has the inequalities:

$$
\begin{aligned}
f\left(\frac{a+b}{2}, \frac{c+d}{2}\right) & \leq \frac{1}{(b-a)(d-c)} \int_{a}^{b} \int_{c}^{d} f(x, y) d y d x \\
& \leq \frac{f(a, c)+s f(b, c)+s f(a, d)+s^{2} f(b, d)}{(s+1)^{2}}
\end{aligned}
$$

The above inequalities are sharp.

In [23], Sarikaya et al. proved some Hadamard-type inequalities for co-ordinated convex functions as followings:

Theorem 6. Let $f: \Delta \subset \mathbb{R}^{2} \rightarrow \mathbb{R}$ be a partial differentiable mapping on $\Delta:=[a, b] \times$ $[c, d]$ in $\mathbb{R}^{2}$ with $a<b$ and $c<d$. If $\left|\frac{\partial^{2} f}{\partial t \partial s}\right|$ is a convex function on the co-ordinates on $\Delta$, then one has the inequalities:

$$
\begin{aligned}
& \mid \frac{f(a, c)+f(a, d)+f(b, c)+f(b, d)}{4} \\
& \frac{1}{(b-a)(d-c)} \int_{a}^{b} \int_{c}^{d} f(x, y) d x d y-A \mid \\
& \leq \frac{(b-a)(d-c)}{16}\left(\frac{\left|\frac{\partial^{2} f}{\partial t \partial s}\right|(a, c)+\left|\frac{\partial^{2} f}{\partial t \partial s}\right|(a, d)+\left|\frac{\partial^{2} f}{\partial t \partial s}\right|(b, c)+\left|\frac{\partial^{2} f}{\partial t \partial s}\right|(b, d)}{4}\right)
\end{aligned}
$$

where

$$
A=\frac{1}{2}\left[\frac{1}{(b-a)} \int_{a}^{b}[f(x, c)+f(x, d)] d x+\frac{1}{(d-c)} \int_{c}^{d}[f(a, y) d y+f(b, y)] d y\right] .
$$

Theorem 7. Let $f: \Delta \subset \mathbb{R}^{2} \rightarrow \mathbb{R}$ be a partial differentiable mapping on $\Delta:=[a, b] \times$ $[c, d]$ in $\mathbb{R}^{2}$ with $a<b$ and $c<d$. If $\left|\frac{\partial^{2} f}{\partial t \partial s}\right|^{q}, q>1$, is a convex function on the co-ordinates on $\Delta$, then one has the inequalities:

$$
\begin{aligned}
& \mid \frac{f(a, c)+f(a, d)+f(b, c)+f(b, d)}{4} \\
& \frac{1}{(b-a)(d-c)} \int_{a}^{b} \int_{c}^{d}(x, y) d x d y-A \mid \\
& \leq \frac{(b-a)(d-c)}{4(p+1) \frac{2}{p}}\left(\frac{\left|\frac{\partial^{2} f}{\partial t \partial s}\right|^{q}(a, c)+\left|\frac{\partial^{2} f}{\partial t \partial s}\right|^{q}(a, d)+\left|\frac{\partial^{2} f}{\partial t \partial s}\right|^{q}(b, c)+\left|\frac{\partial^{2} f}{\partial t \partial s}\right|^{q}(b, d)}{4}\right)^{\frac{1}{q}}
\end{aligned}
$$

where

$$
A=\frac{1}{2}\left[\frac{1}{(b-a)} \int_{a}^{b}[f(x, c)+f(x, d)] d x+\frac{1}{(d-c)} \int_{c}^{d}[f(a, y) d y+f(b, y)] d y\right]
$$


and $\frac{1}{p}+\frac{1}{q}=1$.

Theorem 8. Let $f: \Delta \subset \mathbb{R}^{2} \rightarrow \mathbb{R}$ be a partial differentiable mapping on $\Delta:=[a, b] \times$ $[c, d]$ in $\mathbb{R}^{2}$ with $a<b$ and $c<d$. If $\left|\frac{\partial^{2} f}{\partial t \partial s}\right|^{q}, q \geq 1$, is a convex function on the co-ordinates on $\Delta$, then one has the inequalities:

$$
\begin{aligned}
& \mid \frac{f(a, c)+f(a, d)+f(b, c)+f(b, d)}{4} \\
& \frac{1}{(b-a)(d-c)} \int_{a}^{b} \int_{c}^{d} f(x, y) d x d y-A \mid \\
& \leq \frac{(b-a)(d-c)}{16}\left(\frac{\left|\frac{\partial^{2} f}{\partial t \partial s}\right|^{q}(a, c)+\left|\frac{\partial^{2} f}{\partial t \partial s}\right|^{q}(a, d)+\left|\frac{\partial^{2} f}{\partial t \partial s}\right|^{q}(b, c)+\left|\frac{\partial^{2} f}{\partial t \partial s}\right|^{q}(b, d)}{4}\right)^{\frac{1}{q}}
\end{aligned}
$$

where

$$
A=\frac{1}{2}\left[\frac{1}{(b-a)} \int_{a}^{b}[f(x, c)+f(x, d)] d x+\frac{1}{(d-c)} \int_{c}^{d}[f(a, y) d y+f(b, y)] d y\right]
$$

For refinements, counterparts, generalizations and new Hadamard-type inequalities see the articles [3,5,10-13,19-28]. In [25] (see also [22]), Alomari and Darus introduced new classes of $s$-convex functions on the co-ordinates as following:

Definition 7. Consider the bidimensional interval $\Delta=:[a, b] \times[c, d]$ in $[0, \infty)^{2}$ with $a<b$ and $c<d$. The mapping $f: \Delta \rightarrow \mathbb{R}$ is s-convex in the first sense on $\Delta$ if there exist $s_{1}, s_{2} \in(0,1]$ such that $s=\frac{s_{1}+s_{2}}{2}$,

$$
f(\alpha x+\beta z, \alpha y+\beta w) \leq \alpha^{s_{1}} f(x, y)+\beta^{s_{2}} f(z, w)
$$

holds for all $(x, y),(z, w) \in \Delta$ with $\alpha, \beta \geq 0$ with $\alpha^{s_{1}}+\beta^{s_{2}}=1$ and for some fixed $s_{1}, s_{2}$ $\in(0,1]$. We denote this class of functions by $\mathrm{MWO}_{s_{1}, s_{2}}^{1}$.

Definition 8. Consider the bidimensional interval $\Delta=:[a, b] \times[c, d]$ in $[0, \infty)^{2}$ with $a<b$ and $c<d$. The mapping $f: \Delta \rightarrow \mathbb{R}$ is s-convex in the second sense on $\Delta$ if there exist $s_{1}, s_{2} \in(0,1]$ such that $s=\frac{s_{1}+s_{2}}{2}$,

$$
f(\alpha x+\beta z, \alpha y+\beta w) \leq \alpha^{s_{1}} f(x, y)+\beta^{s_{2}} f(z, w)
$$

holds for all $(x, y),(z, w) \in \Delta$ with $\alpha, \beta \geq 0$ with $\alpha+\beta=1$ and for all fixed $s_{1}, s_{2} \in$ $(0,1]$. We denote this class of functions by $\mathrm{MWO}_{s_{1}, s_{2}}^{2}$.

In [26], Pachpatte established some inequalities for product of convex functions as follows:

Theorem 9. Let $f, g:[a, b] \subseteq \mathbb{R} \rightarrow[0, \infty)$ be convex functions on $[a, b], a<b$.

Then

$$
\frac{1}{b-a} \int_{a}^{b} f(x) g(x) d x \leq \frac{1}{3} M(a, b)+\frac{1}{6} N(a, b)
$$


and

$$
2 f\left(\frac{a+b}{2}\right) g\left(\frac{a+b}{2}\right) \leq \frac{1}{b-a} \int_{a}^{b} f(x) g(x) d x+\frac{1}{6} M(a, b)+\frac{1}{3} N(a, b)
$$

where $M(a, b)=f(a) g(a)+f(b) g(b)$ and $N(a, b)=f(a) g(b)+f(b) g(a)$.

Similar results for $s$-convex functions is due to Kirmaci et al. [13] as follows:

Theorem 10. Let $f, g:[a, b] \subseteq \mathbb{R} \rightarrow \mathbb{R} a, b \in[0, \infty), a<b$, be functions such that $g$ and $f g$ are in $L_{1}([a, b])$. If $f$ is convex and non-negative on $[a, b]$ and if $g$ is s-convex on $[a, b]$, for some $s \in(0,1)$, then

$$
\begin{aligned}
& 2^{s} f\left(\frac{a+b}{2}\right) g\left(\frac{a+b}{2}\right)-\frac{1}{b-a} \int_{a}^{b} f(x) g(x) d x \\
& \leq \frac{1}{(s+1)(s+2)} M(a, b)+\frac{1}{s+2} N(a, b)
\end{aligned}
$$

where $M(a, b)=f(a) g(a)+f(b) g(b)$ and $N(a, b)=f(a) g(b)+f(b) g(a)$.

Theorem 11. Let $f, g:[a, b] \subseteq \mathbb{R} \rightarrow \mathbb{R} a, b \in[0, \infty), a<b$, be functions such that $g$ and $f g$ are in $L_{1}([a, b])$. If $f$ is convex and non-negative on $[a, b]$ and if $g$ is s-convex on $[a, b]$ for some $s \in(0,1)$, then

$$
\frac{1}{b-a} \int_{a}^{b} f(x) g(x) d x \leq \frac{1}{s+2} M(a, b)+\frac{1}{(s+1)(s+2)} N(a, b)
$$

where $M(a, b)=f(a) g(a)+f(b) g(b)$ and $N(a, b)=f(a) g(b)+f(b) g(a)$.

Theorem 12. Let $f, g:[a, b] \subseteq \mathbb{R} \rightarrow \mathbb{R} a, b \in[0, \infty), a<b$, be functions such that $f, g$ and $f g$ are in $L_{1}([a, b])$. If $f$ is $s_{1}$-convex and $g$ is $s_{2}$-convex on $[a, b]$ for some fixed $s_{1}, s_{2}$ $\in(0,1)$, then

$$
\begin{aligned}
\frac{1}{b-a} \int_{a}^{b} f(x) g(x) d x & \leq \frac{1}{s_{1}+s_{2}+1} M(a, b)+B\left(s_{1}+1, s_{2}+1\right) N(a, b) \\
& =\frac{1}{s_{1}+s_{2}+1}\left[M(a, b)+s_{1} s_{2} \frac{\Gamma\left(s_{1}\right) \Gamma\left(s_{2}\right)}{\Gamma\left(s_{1}+s_{2}+1\right)} N(a, b)\right]
\end{aligned}
$$

where $M(a, b)=f(a) g(a)+f(b) g(b)$ and $N(a, b)=f(a) g(b)+f(b) g(a)$.

In the last theorem Beta function of Euler type, defined by

$$
B(x, y)=\int_{0}^{t} t^{x-1}(1-t)^{y-1} d t=\frac{\Gamma(x) \Gamma(y)}{\Gamma(x+y)}
$$

has been used.

The main purpose of the present article is to establish new Hadamard-type inequalities similar to the above inequalities, but now for product of $s$-convex functions (in the second sense) on the co-ordinates in a rectangle from the plane $\mathbb{R}^{2}$.

\section{Main results}

We will start with the following theorem.

Theorem 13. Let $f, g: \Delta=[a, b] \times[c, d] \subseteq[0, \infty)^{2} \rightarrow \mathbb{R}, a<b, c<d$, be functions such that $f, g$ and $f g$ are in $L^{2}(\Delta)$. If $f$ is non-negative and convex on the coordinates on $\Delta$ and if $g$ is s-convex in the second sense on the co-ordinates on $\Delta$, for all $s_{1}, s_{2} \in(0$, $1)$, such that $s=\frac{s_{1}+s_{2}}{2}$, then one has the inequality; 


$$
\begin{aligned}
& \frac{1}{(b-a)(d-c)} \int_{a}^{b} \int_{c}^{d} f(x, y) g(x, y) d y d x \\
& \leq \frac{1}{2}\left(p^{2}+r^{2}\right) L(a, b, c, d)+\frac{1}{2}(p q+r t) M(a, b, c, d)+\frac{1}{2}\left(q^{2}+t^{2}\right) N(a, b, c, d)
\end{aligned}
$$

where

$L(a, b, c, d)=f(a, c) g(a, c)+f(b, c) g(b, c)+f(a, d) g(a, d)+f(b, d) g(b, d)$,

$M(a, b, c, d)=f(a, c) g(a, d)+f(a, d) g(a, c)+f(b, c) g(b, d)+f(b, d) g(b, c)$

$+f(b, c) g(a, c)+f(b, d) g(a, d)+f(a, c) g(b, c)+f(a, d) g(b, d)$,

$N(a, b, c, d)=f(b, c) g(a, d)+f(b, d) g(a, c)+f(a, c) g(b, d)+f(a, d) g(b, c)$

and

$$
p=\frac{1}{s_{2}+2}, \quad q=\frac{1}{\left(s_{2}+1\right)\left(s_{2}+2\right)}, \quad r=\frac{1}{s_{1}+2}, \quad t=\frac{1}{\left(s_{1}+1\right)\left(s_{1}+2\right)} .
$$

Proof. Since $f$ is convex and $g$ is $s$-convex in the second sense on the co-ordinates on $\Delta$. Therefore the partial mappings

$$
f_{y}:[a, b] \rightarrow[0, \infty), \quad f_{y}(x)=f(x, y)
$$

and

$$
f_{x}:[c, d] \rightarrow[0, \infty), \quad f_{x}(y)=f(x, y)
$$

are convex and non-negative on $[a, b]$ and $[c, d]$, respectively. The partial mappings

$$
g_{y}:[a, b] \rightarrow[0, \infty), \quad g_{y}(x)=g(x, y)
$$

and

$$
g_{x}:[c, d] \rightarrow[0, \infty), \quad g_{x}(\gamma)=g(x, y)
$$

are $s_{1^{-}}, s_{2}$-convex on $[a, b]$ and $[c, d]$, respectively, for all $x \in[a, b], y \in[c, d]$, for all $s_{1}, s_{2} \in(0,1]$, such that $s=\frac{s_{1}+s_{2}}{2}$. Now by applying $f_{x}(y) g_{x}(y)$ to $(1.12)$ on $[c, d]$, we get

$$
\begin{aligned}
\frac{1}{d-c} \int_{c}^{d} f_{x}(\gamma) g_{x}(\gamma) d y & \leq p\left[f_{x}(c) g_{x}(c)+f_{x}(d) g_{x}(d)\right] \\
& +q\left[f_{x}(c) g_{x}(d)+f_{x}(d) g_{x}(c)\right] .
\end{aligned}
$$

That is

$$
\begin{aligned}
\frac{1}{d-c} \int_{c}^{d} f(x, y) g(x, y) d y & \leq p[f(x, c) g(x, c)+f(x, d) g(x, d)] \\
& +q[f(x, c) g(x, d)+f(x, d) g(x, c)] .
\end{aligned}
$$

Integrating over $[a, b]$ with respect to $x$ and dividing both sides by $b-a$, we have

$$
\begin{aligned}
& \frac{1}{(b-a)(d-c)} \int_{a}^{b} \int_{c}^{d} f(x, y) g(x, y) d y d x \\
& \leq p\left[\frac{1}{b-a} \int_{a}^{b} f(x, c) g(x, c) d x+\frac{1}{b-a} \int_{a}^{b} f(x, d) g(x, d) d x\right] \\
& +q\left[\frac{1}{b-a} \int_{a}^{b} f(x, c) g(x, d) d x+\frac{1}{b-a} \int_{a}^{b} f(x, d) g(x, c) d x\right] .
\end{aligned}
$$


Now by applying (1.12) to each integral on right-hand side of (2.2) again, we get

$$
\begin{aligned}
\frac{1}{b-a} \int_{a}^{b} f(x, c) g(x, c) d x & \leq p[f(a, c) g(a, c)+f(b, c) g(b, c)] \\
& +q[f(a, c) g(b, c)+f(b, c) g(a, c)] . \\
\frac{1}{b-a} \int_{a}^{b} f(x, d) g(x, d) d x & \leq p[f(a, d) g(a, d)+f(b, d) g(b, d)] \\
& +q[f(a, d) g(b, d)+f(b, d) g(a, d)] . \\
\frac{1}{b-a} \int_{a}^{b} f(x, c) g(x, d) d x & \leq p[f(a, c) g(a, d)+f(b, c) g(b, d)] \\
& +q[f(a, c) g(b, d)+f(b, c) g(a, d)] . \\
\frac{1}{b-a} \int_{a}^{b} f(x, d) g(x, c) d x & \leq p[f(a, d) g(a, c)+f(b, d) g(b, c)] \\
& +q[f(a, d) g(b, c)+f(b, d) g(a, c)] .
\end{aligned}
$$

On substitution of these inequalities in (2.2), we obtain

$$
\begin{aligned}
& \frac{1}{(b-a)(d-c)} \int_{a}^{b} \int_{c}^{d} f(x, y) g(x, y) d y d x \\
& \leq p^{2} L(a, b, c, d)+p q M(a, b, c, d)+q^{2} N(a, b, c, d) .
\end{aligned}
$$

Similarly, if we apply $f_{y}(x) g_{y}(x)$ to $(1.12)$ on $[a, b]$, we get the following result:

$$
\begin{aligned}
& \frac{1}{(b-a)(d-c)} \int_{a}^{b} \int_{c}^{d} f(x, y) g(x, y) d y d x \\
& \leq r^{2} L(a, b, c, d)+\operatorname{rtM}(a, b, c, d)+t^{2} N(a, b, c, d)
\end{aligned}
$$

Adding the inequalities (2.3), (2.4) and dividing by 2, we get (2.1).

Theorem 14. Let $f, g: \Delta=[a, b] \times[c, d] \subseteq \mathbb{R}^{2} \rightarrow \mathbb{R}, a<b, c<d$, be functions such that $f, g$, and $f g$ are in $L^{2}(\Delta)$. If $f$ is non-negative and convex on the co-ordinates on $\Delta$ and if $g$ is s-convex on the co-ordinates on $\Delta$, for all $s_{1}, s_{2} \in(0,1)$, such that $s=\frac{s_{1}+s_{2}}{2}$, then one has the inequality;

$$
\begin{aligned}
2^{s_{1}+s_{2}} f\left(\frac{a+b}{2}, \frac{c+d}{2}\right) g\left(\frac{a+b}{2}, \frac{c+d}{2}\right) \leq & \frac{1}{(b-a)(d-c)} \int_{a}^{b} \int_{c}^{d} f(x, y) g(x, y) d y d x \\
& +\frac{1}{2}\left(q^{2}+t^{2}+2 p t+2 q r\right) L(a, b, c, d) \\
& +\frac{1}{2}(p q+r t+2 q t+2 r p) M(a, b, c, d) \\
& +\frac{1}{2}\left(p^{2}+r^{2}+2 p t+2 r q\right) N(a, b, c, d)
\end{aligned}
$$

where $L(a, b, c, d), M(a, b, c, d), N(a, b, c, d), p, q, r$ and $t$ as in Theorem 13.

Proof. Applying $2^{s_{1}} f\left(\frac{a+b}{2}, \frac{c+d}{2}\right) g\left(\frac{a+b}{2}, \frac{c+d}{2}\right)$ to (1.11) and multiplying both sides by $2^{s_{2}}$, we get 


$$
\begin{aligned}
& 2^{s_{1}+s_{2}} f\left(\frac{a+b}{2}, \frac{c+d}{2}\right) g\left(\frac{a+b}{2}, \frac{c+d}{2}\right) \\
& \leq \frac{2^{s_{2}}}{b-a} \int_{a}^{b} f\left(x, \frac{c+d}{2}\right) g\left(x, \frac{c+d}{2}\right) d x \\
& +t\left[2^{s_{2}} f\left(a, \frac{c+d}{2}\right) g\left(a, \frac{c+d}{2}\right)+2^{s_{2}} f\left(b, \frac{c+d}{2}\right) g\left(b, \frac{c+d}{2}\right)\right] \\
& +r\left[2^{s_{2}} f\left(a, \frac{c+d}{2}\right) g\left(b, \frac{c+d}{2}\right)+2^{s_{2}} f\left(b, \frac{c+d}{2}\right) g\left(a, \frac{c+d}{2}\right)\right] .
\end{aligned}
$$

Similarly, by applying $2^{s_{2}} f\left(\frac{a+b}{2}, \frac{c+d}{2}\right) g\left(\frac{a+b}{2}, \frac{c+d}{2}\right)$ to (1.11) and multiplying both sides by $2^{s_{1}}$, we get

$$
\begin{aligned}
& 2^{s_{1}+s_{2}} f\left(\frac{a+b}{2}, \frac{c+d}{2}\right) g\left(\frac{a+b}{2}, \frac{c+d}{2}\right) \\
& \leq \frac{2^{s_{1}}}{d-c} \int_{c}^{d} f\left(\frac{a+b}{2}, y\right) g\left(\frac{a+b}{2}, y\right) d y \\
& +q\left[2^{s_{1}} f\left(\frac{a+b}{2}, c\right) g\left(\frac{a+b}{2}, c\right)+2^{s_{1}} f\left(\frac{a+b}{2}, d\right) g\left(\frac{a+b}{2}, d\right)\right] \\
& +p\left[2^{s_{1}} f\left(\frac{a+b}{2}, c\right) g\left(\frac{a+b}{2}, d\right)+2^{s_{1}} f\left(\frac{a+b}{2}, d\right) g\left(\frac{a+b}{2}, c\right)\right] .
\end{aligned}
$$

Adding (2.6) and (2.7), we have

$$
\begin{aligned}
& 2^{s_{1}+s_{2}+1} f\left(\frac{a+b}{2}, \frac{c+d}{2}\right) g\left(\frac{a+b}{2}, \frac{c+d}{2}\right) \\
& \leq \frac{2^{s_{2}}}{b-a} \int_{a}^{b} f\left(x, \frac{c+d}{2}\right) g\left(x, \frac{c+d}{2}\right) d x+\frac{2^{s_{1}}}{d-c} \int_{c}^{d} f\left(\frac{a+b}{2}, y\right) g\left(\frac{a+b}{2}, y\right) d y \\
& +t\left[2^{s_{2}} f\left(a, \frac{c+d}{2}\right) g\left(a, \frac{c+d}{2}\right)+2^{s_{2}} f\left(b, \frac{c+d}{2}\right) g\left(b, \frac{c+d}{2}\right)\right] \\
& +r\left[2^{s_{2}} f\left(a, \frac{c+d}{2}\right) g\left(b, \frac{c+d}{2}\right)+2^{s_{2}} f\left(b, \frac{c+d}{2}\right) g\left(a, \frac{c+d}{2}\right)\right] \\
& +q\left[2^{s_{1}} f\left(\frac{a+b}{2}, c\right) g\left(\frac{a+b}{2}, c\right)+2^{s_{1}} f\left(\frac{a+b}{2}, d\right) g\left(\frac{a+b}{2}, d\right)\right] \\
& +p\left[2^{s_{1}} f\left(\frac{a+b}{2}, c\right) g\left(\frac{a+b}{2}, d\right)+2^{s_{1}} f\left(\frac{a+b}{2}, d\right) g\left(\frac{a+b}{2}, c\right)\right] .
\end{aligned}
$$

Applying (1.11) to each term within the brackets, we get

$$
\begin{aligned}
2^{s_{2}} f\left(a, \frac{c+d}{2}\right) g\left(a, \frac{c+d}{2}\right) & \leq \frac{1}{d-c} \int_{c}^{d} f(a, y) g(a, y) d y \\
& +t[f(a, c) g(a, c)+f(a, d) g(a, d)] \\
& +r[f(a, c) g(a, d)+f(a, d) g(a, c)] . \\
2^{s_{2}} f\left(b, \frac{c+d}{2}\right) g\left(b, \frac{c+d}{2}\right) \leq & \frac{1}{d-c} \int_{c}^{d} f(b, y) g(b, y) d y \\
& +t[f(b, c) g(b, c)+f(b, d) g(b, d)] \\
& +r[f(b, c) g(b, d)+f(b, d) g(b, c)] . \\
2^{s_{2}} f\left(a, \frac{c+d}{2}\right) g\left(b, \frac{c+d}{2}\right) \leq & \frac{1}{d-c} \int_{c}^{d} f(a, y) g(b, y) d y \\
& +t[f(a, c) g(b, c)+f(a, d) g(b, d)] \\
& +r[f(a, c) g(b, d)+f(a, d) g(b, c)] . \\
2^{s_{2}} f\left(b, \frac{c+d}{2}\right) g\left(a, \frac{c+d}{2}\right) & \leq \frac{1}{d-c} \int_{c}^{d} f(b, y) g(a, y) d y \\
& +t[f(b, c) g(a, c)+f(b, d) g(a, d)] \\
& +r[f(b, c) g(a, d)+f(b, d) g(a, c)] .
\end{aligned}
$$




$$
\begin{aligned}
2^{s_{1}} f\left(\frac{a+b}{2}, c\right) g\left(\frac{a+b}{2}, c\right) & \leq \frac{1}{b-a} \int_{a}^{b} f(x, c) g(x, c) d x \\
& +q[f(a, c) g(a, c)+f(b, c) g(b, c)] \\
& +p[f(a, c) g(b, c)+f(b, c) g(a, c)] . \\
2^{s_{1}} f\left(\frac{a+b}{2}, d\right) g\left(\frac{a+b}{2}, d\right) \leq & \frac{1}{b-a} \int_{a}^{b} f(x, d) g(x, d) d x \\
& +q[f(a, d) g(a, d)+f(b, d) g(b, d)] \\
& +p[f(a, d) g(b, d)+f(b, d) g(a, d)] . \\
2^{s_{1}} f\left(\frac{a+b}{2}, c\right) g\left(\frac{a+b}{2}, d\right) & \leq \frac{1}{b-a} \int_{a}^{b} f(x, c) g(x, d) d x \\
& +q[f(a, c) g(a, d)+f(b, c) g(b, d)] \\
& +p[f(a, c) g(b, d)+f(b, c) g(a, d)] . \\
2^{s_{1}} f\left(\frac{a+b}{2}, d\right) g\left(\frac{a+b}{2}, c\right) & \leq \frac{1}{b-a} \int_{a}^{b} f(x, d) g(x, c) d x \\
& +q[f(a, d) g(a, c)+f(b, d) g(b, c)] \\
& +p[f(a, d) g(b, c)+f(b, d) g(a, c)] .
\end{aligned}
$$

Substituting these inequalities in (2.8) and simplifying, we obtain

$$
\begin{aligned}
& 2^{s_{1}+s_{2}+1} f\left(\frac{a+b}{2}, \frac{c+d}{2}\right) g\left(\frac{a+b}{2}, \frac{c+d}{2}\right) \\
& \leq \frac{2^{s_{2}}}{b-a} \int_{a}^{b} f\left(x, \frac{c+d}{2}\right) g\left(x, \frac{c+d}{2}\right) d x \\
& +\frac{2^{s_{1}}}{d-c} \int_{c}^{d} f\left(\frac{a+b}{2}, y\right) g\left(\frac{a+b}{2}, y\right) d y \\
& +t \frac{1}{d-c} \int_{c}^{d} f(a, y) g(a, y) d y+t \frac{1}{d-c} \int_{c}^{d} f(b, y) g(b, y) d y \\
& +r \frac{1}{d-c} \int_{c}^{d} f(a, y) g(b, y) d y+r \frac{1}{d-c} \int_{c}^{d} f(b, y) g(a, y) d y \\
& +q \frac{1}{b-a} \int_{a}^{b} f(x, c) g(x, c) d x+q \frac{1}{b-a} \int_{a}^{b} f(x, d) g(x, d) d x \\
& +p \frac{1}{b-a} \int_{a}^{b} f(x, c) g(x, d) d x+p \frac{1}{b-a} \int_{a}^{b} f(x, d) g(x, c) d x \\
& +\left(q^{2}+t^{2}\right) L(a, b, c, d)+(p q+r t) M(a, b, c, d)+\left(p^{2}+r^{2}\right) N(a, b, c, d) .
\end{aligned}
$$

Now by applying $2^{s_{1}} f\left(\frac{a+b}{2}, y\right) g\left(\frac{a+b}{2}, y\right)$ to (1.11), integrating over $[c, d]$ and dividing both sides by $d-c$, we get

$$
\begin{aligned}
& \frac{2^{s_{1}}}{d-c} \int_{c}^{d} f\left(\frac{a+b}{2}, y\right) g\left(\frac{a+b}{2}, y\right) d y \\
& \leq \frac{1}{(b-a)(d-c)} \int_{a}^{b} \int_{c}^{d} f(x, y) g(x, y) d x d y \\
& +t \frac{1}{d-c} \int_{c}^{d} f(a, y) g(a, y) d y+t \frac{1}{d-c} \int_{c}^{d} f(b, y) g(b, y) d y \\
& +r \frac{1}{d-c} \int_{c}^{d} f(a, y) g(b, y) d y+r \frac{1}{d-c} \int_{c}^{d} f(b, y) g(a, y) d y .
\end{aligned}
$$


Again by applying $2^{s_{2}} f\left(x, \frac{c+d}{2}\right) g\left(x, \frac{c+d}{2}\right)$ to (1.11), integrating over $[a, b]$ and dividing both sides by $b$ - $a$, we get

$$
\begin{aligned}
& \frac{2^{s_{2}}}{b-a} \int_{a}^{b} f\left(x, \frac{c+d}{2}\right) g\left(x, \frac{c+d}{2}\right) d x \\
& \leq \frac{1}{(b-a)(d-c)} \int_{a}^{b} \int_{c}^{d} f(x, y) g(x, y) d y d x \\
& +q \frac{1}{b-a} \int_{a}^{b} f(x, c) g(x, c) d x+q \frac{1}{b-a} \int_{a}^{b} f(x, d) g(x, d) d x \\
& +p \frac{1}{b-a} \int_{a}^{b} f(x, c) g(x, d) d x+p \frac{1}{b-a} \int_{a}^{b} f(x, d) g(x, c) d x .
\end{aligned}
$$

Adding (2.10) and (2.11), we have

$$
\begin{aligned}
& \frac{2^{s_{2}}}{b-a} \int_{c}^{d} f\left(x, \frac{c+d}{2}\right) g\left(x, \frac{c+d}{2}\right) d x+\frac{2^{s_{1}}}{d-c} \int_{c}^{d} f\left(\frac{a+b}{2}, y\right) g\left(\frac{a+b}{2}, y\right) d y \\
& \leq \frac{2}{(b-a)(d-c)} \int_{a}^{b} \int_{c}^{d} f(x, y) g(x, y) d y d x \\
& +t \frac{1}{d-c} \int_{c}^{d} f(a, y) g(a, y) d y+t \frac{1}{d-c} \int_{c}^{d} f(b, y) g(b, y) d y \\
& +r \frac{1}{d-c} \int_{c}^{d} f(a, y) g(b, y) d y+r \frac{1}{d-c} \int_{c}^{d} f(b, y) g(a, y) d y \\
& +q \frac{1}{b-a} \int_{a}^{b} f(x, c) g(x, c) d x+q \frac{1}{b-a} \int_{a}^{b} f(x, d) g(x, d) d x \\
& +p \frac{1}{b-a} \int_{a}^{b} f(x, c) g(x, d) d x+p \frac{1}{b-a} \int_{a}^{b} f(x, d) g(x, c) d x .
\end{aligned}
$$

Therefore from (2.9) and (2.12), we obtain

$$
\begin{aligned}
& 2^{s_{1}+s_{2}+1} f\left(\frac{a+b}{2}, \frac{c+d}{2}\right) g\left(\frac{a+b}{2}, \frac{c+d}{2}\right) \\
& \leq \frac{2}{(b-a)(d-c)} \int_{a}^{b} f(x, y) g(x, y) d x d y \\
& +2 t \frac{1}{d-c} \int_{c}^{d} f(a, y) g(a, y) d y+2 t \frac{1}{d-c} \int_{c}^{d} f(b, y) g(b, y) d y \\
& +2 r \frac{1}{d-c} \int_{c}^{d} f(a, y) g(b, y) d y+2 r \frac{1}{d-c} \int_{c}^{d} f(b, y) g(a, y) d y \\
& +2 q \frac{1}{b-a} \int_{a}^{b} f(x, c) g(x, c) d x+2 q \frac{1}{b-a} \int_{a}^{b} f(x, d) g(x, d) d x \\
& +2 p \frac{1}{b-a} \int_{a}^{b} f(x, c) g(x, d) d x+2 p \frac{1}{b-a} \int_{a}^{b} f(x, d) g(x, c) d x \\
& +\left(q^{2}+t^{2}\right) L(a, b, c, d)+(p q+r t) M(a, b, c, d)+\left(p^{2}+r^{2}\right) N(a, b, c, d) .
\end{aligned}
$$


By applying (1.12) to each of the integral in (2.13) and simplifying, we get

$$
\begin{aligned}
& 2^{s_{1}+s_{2}+1} f\left(\frac{a+b}{2}, \frac{c+d}{2}\right) g\left(\frac{a+b}{2}, \frac{c+d}{2}\right) \\
& \leq \frac{2}{(b-a)(d-c)} \int_{a}^{b} \int_{c}^{d} f(x, y) g(x, y) d y d x \\
& +\left(q^{2}+t^{2}+2 p t+2 q r\right) L(a, b, c, d)+(p q+r t+2 q t+2 r p) M(a, b, c, d) \\
& +\left(p^{2}+r^{2}+2 p t+2 r q\right) N(a, b, c, d)
\end{aligned}
$$

Dividing both sides by 2 , we get required result. $\quad \square$

Theorem 15. Let $f, g: \Delta=[a, b] \times[c, d] \subseteq \mathbb{R}^{2} \rightarrow \mathbb{R}, a<b, c<d$, be functions such that $f, g$, and $f g$ are in $L_{2}(\Delta)$. If $f$ is $s_{1}$-convex on the co-ordinates on $\Delta$ and $g$ is $s_{2}$-convex on the co-ordinates on $\Delta$, for some fixed $s_{11}, s_{12}, s_{21}, s_{22} \in(0,1)$, such that $s_{1}=\frac{s_{11}+s_{12}}{2}, s_{2}=\frac{s_{21}+s_{22}}{2}$, then one has the following inequality;

$$
\begin{aligned}
& \frac{1}{(b-a)(d-c)} \int_{a}^{b} \int_{c}^{d} f(x, y) g(x, y) d y d x \\
& \leq \frac{1}{2}\left(p_{1}^{2}+r_{1}^{2}\right) L(a, b, c, d)+\frac{1}{2}\left(p_{1} q_{1}+r_{1} t_{1}\right) M(a, b, c, d)+\frac{1}{2}\left(q_{1}^{2}+t_{1}^{2}\right) N(a, b, c, d)
\end{aligned}
$$

where $L(a, b, c, d), M(a, b, c, d)$, and $N(a, b, c, d)$ as defined in Theorem 13 and $p_{1}=\frac{1}{s_{12}+s_{22}+1}, q_{1}=B\left(s_{12}+1, s_{22}+1\right), r_{1}=\frac{1}{s_{11}+s_{21}+1}, t_{1}=B\left(s_{11}+1, s_{21}+1\right)$.

Proof. By a similar way to Theorem 13 with $p_{1}=\frac{1}{s_{12}+s_{22}+1}, q_{1}=B\left(s_{12}+1, s_{22}+1\right)$, $r_{1}=\frac{1}{s_{11}+s_{21}+1}, t_{1}=B\left(s_{11}+1, s_{21}+1\right)$ and thus (2.14) is established.

Theorem 16. Let $f, g: \Delta=[a, b] \times[c, d] \subseteq \mathbb{R}^{2} \rightarrow \mathbb{R}, a<b, c<d$, be functions such that $f, g$, and $f g$ are in $L_{2}(\Delta)$. If $f$ is $s_{1}$-convex on the co-ordinates on $\Delta$ and $g$ is $s_{2}$-convex on the co-ordinates on $\Delta$, for some fixed $s_{11}, s_{12}, s_{21}, s_{22} \in(0,1)$, such that $s_{1}=\frac{s_{11}+s_{12}}{2}, s_{2}=\frac{s_{21}+s_{22}}{2}$, then one has the following inequality;

$$
\begin{aligned}
& 2^{s_{11}+s_{21}+s_{12}+s_{22}-2} f\left(\frac{a+b}{2}, \frac{c+d}{2}\right) g\left(\frac{a+b}{2}, \frac{c+d}{2}\right) \\
& \leq \frac{1}{(b-a)(d-c)} \int_{a}^{b} \int_{c}^{d} f(x, y) g(x, y) d y d x \\
& +\frac{1}{2}\left(q_{1}^{2}+t_{1}^{2}+2 p_{1} t_{1}+2 q_{1} r_{1}\right) L(a, b, c, d) \\
& +\frac{1}{2}\left(p_{1} q_{1}+r_{1} t_{1}+2 q_{1} t_{1}+2 r_{1} p_{1}\right) M(a, b, c, d) \\
& +\frac{1}{2}\left(p_{1}^{2}+r_{1}^{2}+2 p_{1} t_{1}+2 r_{1} q_{1}\right) N(a, b, c, d)
\end{aligned}
$$

where $L(a, b, c, d), M(a, b, c, d)$, and $N(a, b, c, d)$ as defined in Theorem 13 and $p_{1}=\frac{1}{s_{12}+s_{22}+1}, q_{1}=B\left(s_{12}+1, s_{22}+1\right), r_{1}=\frac{1}{s_{11}+s_{21}+1}, t_{1}=B\left(s_{11}+1, s_{21}+1\right)$.

Proof. By a similar way to Theorem 13 with $p_{1}=\frac{1}{s_{12}+s_{22}+1}, q_{1}=B\left(s_{12}+1, s_{22}+1\right)$, $r_{1}=\frac{1}{s_{11}+s_{21}+1}, t_{1}=B\left(s_{11}+1, s_{21}+1\right)$ the proof is completed

\section{Author details}

${ }^{1}$ Department of Mathematics, K.K. Education Faculty, Ataturk University, Erzurum 25240, Turkey ${ }^{2}$ Department of Mathematics, College of Science, University of Hail, Hail 2440, Saudi Arabia ${ }^{3}$ Department of Mathematics, Faculty of Science and Letters, Ağri İbrahim Çeçen University, Ağri 04100, Turkey 


\section{Authors' contributions}

MA and AOA carried out the design of the study and performed the analysis. MEO (adviser) participated in its design and coordination. All authors read and approved the final manuscript.

\section{Competing interests}

The authors declare that they have no competing interests.

Received: 4 May 2011 Accepted: 5 February 2012 Published: 5 February 2012

\section{References}

1. Godunova, EK, Levin, VI: Neravenstva dlja funkcii širokogo klassa, soderžašcego vypuklye, monotonnye i nekotorye drugie vidy funkcii. Vycislitel Mat i Mat Fiz Mežvuzov Sb Nauc Trudov, MGPI, Moskva. 138-142 (1985)

2. Mitrinović, DS, Pečarić, J, Fink, AM: Classical and New Inequalities in Analysis. Kluwer Academic Publishers, Dordrecht (1993)

3. Dragomir, SS, Pečarić, J, Persson, LE: Some inequalities of Hadamard type. Soochow J Math. 21, 335-341 (1995)

4. Breckner, WW: Stetigkeitsaussagen für eine klasse verallgemeinerter konvexer funktionen in topologischen linearen Raumen. Publ Inst Math. 23, 13-20 (1978)

5. Hudzik, H, Maligranda, L: Some remarks on s-convex functions. Aequationes Math. 48, 100-111 (1994). doi:10.1007/ BF01837981

6. Breckner, WW: Continuity of generalized convex and generalized concave set-valued functions. Rev Anal Numér Theor Approx. 22, 39-51 (1993)

7. Orlicz, W: A note on modular spaces. I Bull Acad Polon Sci Math Astronom Phys. 9, 157-162 (1961)

8. Matuszewska, W, Orlicz, W: A note on the theory of s-normed spaces of $\varphi$-integrable functions. Studia Math. 21, 107-115 (1981)

9. Musielak, J: Orlicz spaces and modular spaces. In Lecture Notes in Mathematics, vol. 1034,Springer-Verlag, Berlin (1983)

10. Dragomir, SS, Fitzpatrick, S: The Hadamard's inequality for s-convex functions in the second sense. Demonstratio Math. 32(4):687-696 (1999)

11. Dragomir, SS, Pearce, CEM: Selected topics on Hermite-Hadamard inequalities and applications. RGMIA monographs, Victoria University. http://www.staff.vu.edu.au/RGMIA/monographs/hermite-hadamard.html (2000)

12. Hussain, S, Bhatti, Ml, Iqbal, M: Hadamard-type inequalities for s-convex functions. Punjab Univ J Math. 41, 51-60 (2009)

13. Kirmaci, US, Bakula, MK, Özdemir, ME, Pečarić, J: Hadamard-type inequalities for s-convex functions. Appl Math Comput. 193, 26-35 (2007). doi:10.1016/j.amc.2007.03.030

14. Varošanec, S: On h-convexity. J Math Anal Appl. 326, 303-311 (2007). doi:10.1016/j.jmaa.2006.02.086

15. Bombardelli, $M$, Varošanec, S: Properties of $h$-convex functions related to the Hermite-Hadamard-Fejér inequalities. Comput Math Appl. 58, 1869-1877 (2009). doi:10.1016/j.camwa.2009.07.073

16. Burai, P, Hazy, A: On approximately $h$-convex functions. J Convex Anal. 18(2):447-454 (2011)

17. Sarikaya, MZ, Sağlam, A, Yildirim, H: On some Hadamard-type inequalities for $h$-convex functions. J Math Inequal. 2(3):335-341 (2008)

18. Sarikaya, MZ, Set, E, Özdemir, ME: On some new inequalities of Hadamard type involving $h$-convex functions. Acta Math Univ Comenianae. LXXIX(2):265-272 (2010)

19. Dragomir, SS: On Hadamard's inequality for convex functions on the co-ordinates in a rectangle from the plane. Taiwanese J Math. 5, 775-788 (2001)

20. Alomari, M, Darus, $\mathrm{M}$ : The Hadamard's inequality for s-convex function of 2-variables on the co-ordinates. Int J Math Anal. 2(13):629-638 (2008)

21. Alomari, M, Darus, M: Co-ordinated s-convex function in the first sense with some Hadamard-type inequalities. Int J Contemp Math Sci. 32, 1557-1567 (2008)

22. Alomari, M, Darus, M: Hadamard-type inequalities for s-convex functions. Int Math Forum. 3(40):1965-1975 (2008)

23. Sarikaya, MZ, Set, E, Özdemir, ME, Dragomir, SS: New some Hadamard's type inequalities for co-ordinated convex functions. Tamsui Oxford J Math. (2011, in press)

24. Alomari, M, Darus, M: The Hadamard's inequality for s-convex function. Int J Math Anal. 2(13):639-646 (2008)

25. Alomari, M, Darus, M: On co-ordinated s-convex functions. Int Math Forum. 3(40):1977-1989 (2008)

26. Pachpatte, BG: On some inequalities for convex functions. RGMIA Res Rep Coll. 6(E)http://ajmaa.org/RGMIA/ monographs/hermite_hadamard.html (2003)

27. Özdemir, ME, Set, E, Sarikaya, MZ: Some new Hadamard's type inequalities for co-ordinated $m$-convex and $(a, m)$ convex functions. Hacettepe J Math Ist. 40, 219-229 (2011)

28. Akdemir, AO, Ozdemir, ME: Some Hadamard-type inequalities for co-ordinated P-convex functions and Godunova-Levin functions. In AlP Conference Proceedings, vol. 1309, pp. 7-15.American Institute of Physics, Melville, New York (2010)

doi:10.1186/1029-242X-2012-21

Cite this article as: Özdemir et al: On some Hadamard-type inequalities for product of two s-convex functions on the co-ordinates. Journal of Inequalities and Applications 2012 2012:21. 